\title{
The Best Growth and Approximation of Entire Functions of Two Complex Variables in Banach Spaces
}

\author{
Dr.Deepti Goyal, Dr.D.P.Singh \\ RKGIT, Ghaziabad ,DIT School of engineering Gr.Noida
}

\begin{abstract}
In this Paper we are studding the polynomial approximation of entire functions of two complex variables in Banach spaces; concept is depend on index-pair. The characterizations of $(p, q)$-order of entire functions of two complex variables have been studied in terms of approximation errors. The results can be extended to m-variables but to reduce the mechanical labour we have considered only two variables.
\end{abstract}

Key Words: Approximation error, order, type, entire function, index-pair.

\section{Introduction:}

Let $\varphi\left(z_{1} z_{2}\right)=\sum_{m_{1}, m_{2}=0}^{\infty}\left\{a_{m_{1}, m_{2}}\left(z_{1}^{m_{1}}, z_{2}^{m_{2}}\right)\right\}$ be a function of the complex variables $z_{1}$ and $z_{2}$, regular for $\left|z_{1}\right| \leq r_{n} \quad n=1,2$. If $r_{1}$ and $r_{2}$ can be taken arbitrarily large, then $\varphi\left(z_{1} z_{2}\right)$ represents an entire function of complex variables $z_{1}$ and $z_{2}$. Following Bose and Sharma [1] we define the maximum modulus of $\varphi\left(z_{1} z_{2}\right)$ as

$$
M\left(r_{1}, r_{2}\right)=\max _{\left|z_{n}\right| \leq r_{n}}\left|\varphi\left(z_{1} z_{2}\right)\right|, n=1,2
$$

The order $\rho$ of the entire function $\varphi\left(z_{1}, z_{2}\right)$ is defined as [1];

$$
\rho=\lim _{r_{1}, r_{2 \rightarrow \infty}} \sup \frac{\log \log M\left(r_{1}, r_{2}\right)}{\log \left(r_{1}, r_{2}\right)}
$$

Bose and Sharma [1], obtained the following characterizations for order of entire functions of two complex variables.

Theorem 1.1. The entire function $\varphi\left(z_{1 .} z_{2}\right)=\sum_{m_{1}, m_{2}=0}^{\infty}\left\{a_{m_{1}, m_{2}}\left(z_{1}^{m_{1}}, z_{2}^{m_{2}}\right)\right\}$ is of finite order if and only if

$$
\mu=\lim _{m_{1, m_{2 \rightarrow \infty}}} \sup \frac{\log \left(\mathrm{m}_{1} \mathrm{~m}_{1, \mathrm{~m}_{2}} \mathrm{~m}_{2}\right)}{\log \left(\left|a_{m_{1}, m_{2}}\right|^{-1}\right)}
$$

is finite and then the order $\rho$ of $\varphi\left(z_{1} z_{2}\right)$ is equal to $\mu$.

Let $H_{\vartheta}, \vartheta>0$ denote the space of functions $f\left(z_{1} z_{2}\right)$ analytic in the unit bi-disc $U=\left\{z_{1}, z_{1}, \in:\left|z_{1}\right|<1,\left|z_{2}\right|<1\right\}$ Such that $\|\varphi\|_{T_{\vartheta}}=\lim _{r_{1, r_{2}}} M_{\vartheta}\left(\varphi, r_{1}, r_{2}\right)<\infty$,

Where

$$
M_{\vartheta}\left(\varphi, r_{1}, r_{2}\right)=\left\{\frac{1}{4 \pi^{2}} \int_{-\pi}^{\pi} \int_{-\pi}^{\pi}\left|\varphi\left(r_{1} e^{i \theta_{1}}, r_{2} e^{i \theta_{2}}\right)\right|^{\vartheta} d \theta_{1} d \theta_{2}\right\}^{1 / \vartheta}, \text { and let } H_{\vartheta}^{\prime}, \vartheta>0 \text { denote the space of }
$$
functions $f\left(z_{1}, z_{2}\right)$ analytic in $U$ and satisfying the condition

Set

$$
\|\varphi\|_{T_{\vartheta}}=\left\{\frac{1}{\pi^{2}} \int_{\left|z_{1}\right|<1} \int_{\left|z_{2}\right|<1}\left|\varphi\left(z_{1} z_{2}\right)\right|^{\vartheta} d x_{1} d y_{1} d x_{2} d y_{2}\right\}^{1 / \vartheta}<\infty
$$

$\|\varphi\|_{\infty}=\sup \left\{\left|\varphi\left(z_{1} z_{2}\right)\right|: z_{1}, z_{2} \in U\right\}$

$H_{\vartheta}$ and $H_{\vartheta}^{\prime}$, are Banach spaces for $\vartheta \geq 1$ In analogy with spaces of functions of one variable, we call $H_{\vartheta}$ and $H_{\vartheta}^{\prime}$, the Hardy and Bergman spaces respectively.

Following the Vakarchuk and Zhir [4] we say that the function $\varphi\left(z_{1} z_{2}\right)$ analytic in $U$ belongs to the $\operatorname{space} B(u, v, k)$ where $0<u<v \leq \infty$ and $0<k \leq \infty$ if

$$
\begin{gathered}
\|\varphi\|_{u, v, k}=\left\{\int_{0}^{1} \int_{0}^{1}\left\{\left(1-r_{1}\right)\left(1-r_{2}\right)\right\}^{k\left(\frac{1}{u}-\frac{1}{v}\right)^{-1}} M_{v}^{k}\left(\varphi, r_{1}, r_{2}\right) d r_{1} d r_{2}\right\}^{\frac{1}{N}}<\infty \\
\|\varphi\|_{u, v, \infty}=\sup \left\{\left\{\left(1-r_{1}\right)\left(1-r_{2}\right)\right\}^{\left(\frac{1}{u}-\frac{1}{v}\right)^{-1}} M_{v}\left(\varphi, r_{1}, r_{2}\right): 0<r_{1}, r_{2}<1\right\}<\infty
\end{gathered}
$$

The space $B(u, v, k)$ is a Banach space for $u>0$ and $v, k \geq 1$ otherwise it is a Frechet space. Further, we have 


$$
H_{\vartheta} \sqsubset H_{\vartheta}^{\prime}=B\left(\frac{v}{2}, v, v\right), 1 \leq v<\infty .
$$

Let $Y$ is a Banach space and let $E_{m_{1, m_{2}}(\varphi, Y)}$ be the best approximation of a function $\varphi\left(z_{1 .} z_{2}\right) \in Y$ by elements of the space $Q$ that consists of algebraic polynomials of degree $\leq m_{1}+m_{2}$ in two complex variables.

$$
E_{m_{1, m_{2}}(\varphi, Y)}=\inf \left\{\|\varphi-q\|_{Y}: q \in Q\right\} \text {. }
$$

Recently, Ganti and Srivastava [2] characterized the order and type in terms of the approximation errors $E_{m_{1}, m_{2}(\varphi, B(u, v, k))}$ and $E_{m_{1, m_{2}}\left(\varphi, T_{\vartheta}\right)}$. But their results leave to study a big class of entire functions such as slow growth and fast growth. To bridge this gap in this chapter we pick up the concept of $(p, q)$ - order introduced by Juneja et al. [3] and consider it for entire functions of two variables. Roughly speaking, this concept is a modification of the classical definition of order obtained by replacing logarithms by iterated logarithms, where the degrees of iteration are determined by $\mathrm{p}$ and $\mathrm{q}$.

To the best of our knowledge, characterizations for the $(p, q)$-order of entire functions of two complex variables in Banach spaces have not been obtained so far. We define the $(p, q)-$ order of an entire function $\varphi\left(z_{1} z_{2}\right)$ by

$$
(p, q)=\lim _{r_{1}, r_{2} \rightarrow \infty} \sup \frac{\log ^{p} M\left(r_{1}, r_{2}\right)}{\log ^{q}\left(r_{1}, r_{2}\right)}
$$

Where $\mathrm{p}$ and $\mathrm{q}$ are integers such that $p \geq q \geq 1$.

Notations: We are using the following notations in this paper.

$$
\log ^{[m]} x=\exp ^{[-m]} x=\log \left(\log ^{[m-1]} x\right)=\exp \left(\exp ^{[-(m-1)]} x\right), m=0, \pm 1 . \pm 2 \ldots \ldots
$$

Provided that $0<\log ^{[m-1]} x<\infty$ with $\log ^{[0]} x=\exp ^{[0]} x=x$.

(ii) $\quad \beta^{1 / k}\left[(n+1) k+1 ; k\left(\frac{1}{u}-\frac{1}{2}\right)\right]=\beta(n, u, 2, k)$

$$
\begin{aligned}
& \beta^{1 / k}\left[(m+1) k+1 ; k\left(\frac{1}{u}-\frac{1}{2}\right)\right]=\beta(m, u, 2, k) \\
& \beta^{1 / k}\left[(n+1) k+1, k\left(\frac{1}{u}-\frac{1}{v}\right)\right]=\beta(n, u, v, k) \\
& \beta^{1 / k}\left[(m+1) k+1 ; k\left(\frac{1}{u}-\frac{1}{v}\right)\right]=\beta(m, u, v, k)
\end{aligned}
$$

\section{Basic results:}

In this section we have given some lemmas as basic results, which have been used in the sequel.

Lemma 2.1. If $\varphi\left(z_{1} z_{2}\right)=\sum_{m_{1}, m_{2}=0}^{\infty}\left\{a_{m_{1}, m_{2}}\left(z_{1}^{m_{1}}, z_{2}^{m_{2}}\right)\right\} \quad$ be an entire function and for a pair of integers $(p, q), p \geq 2, q \geq 1 \rho(p, q)$ be defined by (1.3) then

Where

$$
\rho(p, q)=P(L(p, q))
$$

$$
L(p, q)=\lim _{m_{1}+m_{2 \rightarrow \infty}} \sup \frac{\log ^{[p-1]}\left\{\left(m_{1}+m_{2}\right) a_{m_{1}, m_{2}}\right\}}{\log ^{[q-1]}\left\{\frac{1}{m_{1}+m_{2}} \log \left|a_{m_{1}, m_{2}}\right|^{-1}\right\}}
$$

And

$$
P(L(p, q))=\left\{\begin{array}{cl}
L(p, q) & \text { if } p>q \\
1+L(p, q) & \text { if } p=q=2 \\
\max (1+L(p, q)) & \text { if } 3 \leq p=q<\infty \\
\infty & \text { if } p=q=\infty
\end{array}\right.
$$

$$
a_{m_{1, m_{2}}}=\left\{\begin{array}{cl}
\left(\mathrm{m}_{1} \mathrm{~m}_{1}, \mathrm{~m}_{2} \mathrm{~m}_{2}\right)^{\frac{1}{m_{1}+m_{2}}} & ; m_{1}, m_{2} \geq 1 \text { for }(p, q)=(2,1) \\
1 & ; m_{1}, m_{2,} \geq 1 \text { for } 2 \leq q \leq p<\infty \\
0 & ; \text { at least one } m_{1}, m_{2,}=0
\end{array}\right.
$$




\section{Main Results: In this section we prove our main results.}

Theorem3.1: If $\varphi\left(z_{1 .} z_{2}\right)=\sum_{m_{1}, m_{2}=0}^{\infty}\left\{a_{m_{1}, m_{2}}\left(z_{1}^{m_{1}}, z_{2}^{m_{2}}\right)\right\}$ be an entire function and for a pair of integers $(p, q), p \geq 2, q \geq 1$, be defined by (1.3) then

$$
\begin{gathered}
\rho(p, q)=P\left(L^{\prime}(p, q)\right) \text { where } \\
L^{\prime}(p, q)=\lim _{m_{1}+m_{2} \rightarrow \infty} \sup \frac{\log [p-1]\left\{\left(m_{1}+m_{2}\right) a_{m_{1}, m_{2}}\right\}}{\log [q-1]\left\{\frac{1}{m_{1}+m_{2}} \log \left[E_{m_{1}, m_{2}}(\varphi, \beta(u, v, k))\right]^{-1}\right\}}
\end{gathered}
$$

Proof. We prove the above result in two steps, first we consider the space $\beta(u, v, k)$, $v=2,0<v<2$ and $k \geq 1$.Let $\varphi\left(z_{1 .} z_{2}\right) \in \beta(u, v, k)$ be of $(p, q)$ order $\rho(p, q)$ From (2.1), for any $\varepsilon>$ 0 there exists a natural number $m_{0}=m_{0}(\varepsilon)$ such that

$\left|a_{m_{1}, m_{2}}\right| \leq\left[\exp ^{[q-1]}\left\{\log ^{[p-2]}\left\{\left(m_{1}+m_{2}\right) a_{m_{1}, m_{2}}\right\}\right\}^{\frac{1}{L^{(p, q)+\varepsilon}}}\right]^{-\left(m_{1}+m_{2}\right)}, m_{1}, m_{2}>m_{0}$

Now first we show that $\rho(p, q) \geq P\left(L^{\prime}(p, q)\right)$.If $L^{\prime}(p, q)=\infty$ then $\rho(p, q)=\infty$ or else $\varphi\left(z_{1} z_{2}\right)$ is not an entire function. If $L^{\prime}(p, q)=0, \rho(p, q) \geq P\left(L^{\prime}(p, q)\right)$ since $\rho(p, q)$ is nonnegative then we have $0<\varepsilon<$ $L^{\prime}(p, q)<\infty$.

We denote the partial sum of the Taylor series of a function $\varphi\left(z_{1} z_{2}\right)$ by

and

$$
H_{m_{1}, m_{2}}\left(\varphi, z_{1 .} z_{2}\right)=\sum_{i_{1}=0}^{m_{1}} \sum_{i_{2}=0}^{m_{2}} a_{i_{1}, i_{2}} z_{1}^{i_{1}}, z_{2}^{i_{2}}
$$

$$
\begin{gathered}
E_{m_{1}, m_{2}}(\varphi, \beta(u, 2, k))=\left\|\varphi-H_{m_{1}, m_{2}}(\varphi)\right\|_{u .2, k} \\
=\left\{\int_{0}^{1} \int_{0}^{1}\left\{\left(1-r_{1}\right)\left(1-r_{2}\right)\right\}^{k\left(\frac{1}{u}-\frac{1}{2}\right)-1}\left(\sum_{i_{1}} \sum_{i_{2}} r_{1}^{2 i_{1}} r_{2}^{2 i_{2}}\left|a_{i_{1}, i_{2}}\right|^{2}\right)^{\frac{k}{2}} d r_{1} d r_{2}\right\}^{\frac{1}{k}},
\end{gathered}
$$

Where

$\sum_{i_{1}} \sum_{i_{2}} r_{1}^{2 i_{1}} r_{2}^{2 i_{2}}\left|a_{i_{1}, i_{2}}\right|^{2}=R_{1}+R_{2}+\sum_{i_{1}=m_{1}+1}^{\infty} \sum_{i_{2}=m_{2}+1}^{\infty} r_{1}^{2 i_{1}} r_{2}^{2 i_{2}}\left|a_{i_{1}, i_{2}}\right|^{2}$,

$R_{1}=\sum_{i_{1}=0}^{m_{1}} \sum_{i_{2}=m_{2}+1}^{\infty} r_{1}^{2 i_{1}} r_{2}^{2 i_{2}}\left|a_{i_{1}, i_{2}}\right|^{2}$ and $\quad R_{2}=\sum_{i_{1}=m_{1}+1}^{\infty} \sum_{i_{2}=0}^{m_{2}} r_{1}^{2 i_{1}} r_{2}^{2 i_{2}}\left|a_{i_{1}, i_{2}}\right|^{2}$

Since $R_{1}, R_{2}$ are bounded and $r_{1}, r_{2}<1$, therefore (3.3) becomes

$E_{m_{1}, m_{2}}(\varphi, \beta(u, 2, k)) \leq C\left[\int_{0}^{1}\left\{(1-r)^{k\left(\frac{1}{u}-\frac{1}{2}\right)-1}\right\} r^{(s+1) k} d r\right]\left\{\sum_{i_{1}=m_{1}+1}^{\infty} \sum_{i_{2}=m_{2}+1}^{\infty}\left|a_{i_{1}, i_{2}}\right|^{2}\right\}^{\frac{1}{2}}$

Where

$$
\begin{aligned}
& {\left[\int_{0}^{1}\left\{(1-r)^{k\left(\frac{1}{u}-\frac{1}{2}\right)-1}\right\} r^{(s+1) k} d r\right]} \\
& =\left[\int_{0}^{1}\left\{\left(1-r_{1}\right)^{k\left(\frac{1}{u}-\frac{1}{2}\right)-1}\right\} r_{1}{ }^{\left(m_{1}+1\right) k} d r_{1}\right] \times\left[\int_{0}^{1}\left\{\left(1-r_{2}\right)^{k\left(\frac{1}{u}-\frac{1}{2}\right)-1}\right\} r_{2}^{\left(m_{2}+1\right) k} d r_{2}\right]
\end{aligned}
$$

Therefore

$E_{m_{1}, m_{2}}(\varphi, \beta(u, 2, k)) \leq C^{\prime} \beta\left(m_{1}, u, 2, k\right) \beta\left(m_{2}, u, 2, k\right)\left\{\sum_{i_{1}=m_{1}+1}^{\infty} \sum_{i_{2}=m_{2}+1}^{\infty}\left|a_{i_{1}, i_{2}}\right|^{2}\right\}^{\frac{1}{2}}$

where $C^{\prime}$ is a constant and $\beta(a, b)(a, b)>0$ denotes the beta function.

In view of (3.2), we have

$$
\begin{gathered}
\sum_{i_{1}=m_{1}+1}^{\infty} \sum_{i_{2}=m_{2}+1}^{\infty}\left|a_{i_{1}, i_{2}}\right|^{2} \leq \sum_{i_{1}=m_{1}+1}^{\infty} \sum_{i_{2}=m_{2}+1}^{\infty}\left\{\exp ^{[q-1]}\left\{\log ^{[p-2]}\left(i_{1}+i_{2}\right)\right\} \frac{1}{\underline{L}^{L}+\varepsilon}\right\}^{-2\left(i_{1}+i_{2}\right)} \\
=O(1)\left[\exp ^{[q-1]}\left\{\log ^{[p-2]}\left(m_{1}+1+m_{2}+1\right)\right\}^{\frac{1}{L^{\prime}(p, q)+\varepsilon}}\right]^{-2\left(m_{1}+1+m_{2}+1\right)}
\end{gathered}
$$


Using the above inequality in (3.4) we get

$E_{m_{1}, m_{2}}(\varphi, \beta(u, 2, k)) \leq C^{\prime \prime} \beta\left(m_{1}, u, 2, k\right) \beta\left(m_{2}, u, 2, k\right)$

$$
\times\left[\exp ^{[q-1]}\left\{\log ^{[p-2]}\left(m_{1}+m_{2}+2\right)\right\}^{\frac{1}{L(p, q)+\varepsilon}}\right]^{-\left(m_{1}+m_{2}+2\right)}
$$

The result for has been obtained by Ganti and Srivastava [2].

Now consider for $(p, q)=(2,2)$

$E_{m_{1}, m_{2}}(\varphi, \beta(u, 2, k)) \leq C^{\prime \prime} \beta\left(m_{1}, u, 2, k\right) \beta\left(m_{2}, u, 2, k\right) \times$

$$
\left[\exp \left\{\left(m_{1}+1+m_{2}+1\right)\right\}^{\frac{1}{L^{\prime}(2,2)+\varepsilon}}\right]^{-\left(m_{1}+1+m_{2}+1\right)}
$$

Or

$$
\begin{aligned}
& \log E_{m_{1}, m_{2}}(\varphi, \beta(u, 2, k))-\log \beta\left(m_{1}, u, 2, k\right)-\log \beta\left(m_{2}, u, 2, k\right) \\
\leq & \log \left[\exp \left\{\left(m_{1}+1+m_{2}+1\right)\right\}^{\frac{1}{L^{\prime}(2,2)+\varepsilon}}\right]^{-\left(m_{1}+1+m_{2}+1\right)}
\end{aligned}
$$

Or

Or

$$
\begin{aligned}
& \log E_{m_{1}, m_{2}}(\varphi, \beta(u, 2, k))-\log \beta\left(m_{1}, u, 2, k\right)-\log \beta\left(m_{2}, u, 2, k\right) \\
\leq & -\left(m_{1}+m_{2}+2\right) \log \left\{\exp \left\{\left(m_{1}+m_{2}+2\right)\right\}^{\frac{1}{\bar{L}^{(2,2)+\varepsilon}}}\right\}
\end{aligned}
$$

$\frac{1}{\log E_{m_{1}, m_{2}}(\varphi, \beta(u, 2, k))-\log \beta\left(m_{1}, u, 2, k\right)-\log \beta\left(m_{2}, u, 2, k\right)} \geq \frac{1}{-\left(m_{1}+m_{2}+2\right) \log \left\{\exp \left\{\left(m_{1}+m_{2}+2\right)\right\}^{\frac{L^{\prime}(2,2)+\varepsilon}{1}}\right\}}$

Or

$$
L^{\prime}(2,2)+\varepsilon \geq \frac{\log \left(m_{1}+m_{2}\right)}{\log \left[-\frac{1}{m_{1}+m_{2}}\left\{\log E_{m_{1}, m_{2}}(\varphi, \beta(u, 2, k))-\log \beta\left(m_{1}, u, 2, k\right)-\log \beta\left(m_{2}, u, 2, k\right)\right\}\right]}
$$

Since

$$
\left\{\beta\left[(n+1) k+1 ; k\left(\frac{1}{u}-\frac{1}{2}\right)\right]\right\}^{1 /(n+1)} \cong 1 .
$$

Now proceeding to limits, we get

Or

$$
L^{\prime}(2,2) \geq \lim _{m_{1}+m_{2} \rightarrow \infty} \sup \frac{\log \left(m_{1}+m_{2}\right)}{\log \log \left[\log E_{m_{1}, m_{2}}(\varphi, \beta(u, 2, k))\right]^{-\frac{1}{\left(m_{1}+m_{2}\right)}}}
$$

$$
P\left(L^{\prime}(2,2)\right)-1 \geq \lim _{m_{1}+m_{2} \rightarrow \infty} \sup \frac{\log \left(m_{1}+m_{2}\right)}{\log \log \left[\log E_{m_{1}, m_{2}}(\varphi, \beta(u, 2, k))\right]^{-\frac{1}{\left(m_{1}+m_{2}\right)}}}
$$

Or

$$
P\left(L^{\prime}(2,2)\right)-1 \geq L^{\prime}(2,2)
$$

8.

Or

$\rho(2,2) \geq P\left(L^{\prime}(2,2)\right) \geq L^{\prime}(2,2)+1$

Now for $(p, q) \neq(2,1)$ and $(2,2)$ we have from $(3.5)$ that

$E_{m_{1}, m_{2}}(\varphi, \beta(u, 2, k)) \leq C^{\prime \prime} \beta\left(m_{1}, u, 2, k\right) \beta\left(m_{2}, u, 2, k\right)$

Or

$$
\times\left[\exp ^{[q-1]}\left\{\log ^{[p-2]}\left(m_{1}+m_{2}+2\right)\right\}^{\frac{1}{L^{(p, q)+\varepsilon}}}\right]^{-\left(m_{1}+m_{2}+2\right)}
$$

$$
\begin{aligned}
& \log E_{m_{1}, m_{2}}(\varphi, \beta(u, 2, k))-\log \beta\left(m_{1}, u, 2, k\right)-\log \beta\left(m_{2}, u, 2, k\right) \\
\leq & -\left(m_{1}+m_{2}+2\right) \log \left[\exp ^{[q-1]}\left\{\log ^{[p-2]}\left(m_{1}+m_{2}+2\right)\right\}^{\frac{1}{L^{\prime}(p, q)+\varepsilon}}\right]
\end{aligned}
$$


Taking (3.6) into account, we get

$\left[E_{m_{1}, m_{2}}(\varphi, \beta(u, 2, k))\right]^{\frac{-1}{\left(m_{1}+m_{2}\right)}} \geq \exp ^{[q-1]}\left\{\log ^{[p-2]}\left(m_{1}+m_{2}\right)\right\}^{\frac{1}{L^{\prime}(p, q)+\varepsilon}}$

Or

$\log ^{[q-1]}\left[E_{m_{1}, m_{2}}(\varphi, \beta(u, 2, k))\right]^{\frac{-1}{\left(m_{1}+m_{2}\right)}} \geq\left\{\log ^{[p-2]}\left(m_{1}+m_{2}\right)\right\}^{\frac{1}{L^{\prime}(p, q)+\varepsilon}}$

Or

$L^{\prime}(p, q)+\varepsilon \geq \frac{\log ^{[p-1]}\left(m_{1}+m_{2}\right)}{\log ^{[q]}\left[E_{m_{1}, m_{2}}(\varphi, \beta(u, 2, k))\right]^{\frac{-1}{\left(m_{1}+m_{2}\right)}}}$

Proceeding to limits, we obtain

$$
L^{\prime}(p, q) \geq \lim _{m_{1}+m_{2} \rightarrow \infty} \sup \frac{\log ^{[p-1]}\left(m_{1}+m_{2}\right)}{\log [q]\left[E_{m_{1}, m_{2}}(\varphi, \beta(u, 2, k))\right]^{\frac{-1}{\left(m_{1}+m_{2}\right)}}}
$$

9.

Combining other results for $(p, q)=(2,1)$ and $(2,2)$ with $(3.8)$ we get

$$
\rho(p, q) \geq P\left(L^{\prime}(p, q)\right)
$$

To prove reverse inequality consider (eq.2.4 [2]) which gives

Or

$$
\left|a_{m_{1}+1, m_{2+1}}\right| \beta\left(m_{1}, u, 2, k\right) \beta\left(m_{2}, u, 2, k\right) \leq E_{m_{1}, m_{2}}(\varphi, \beta(u, 2, k))
$$

$$
\log \left|a_{m_{1}+1, m_{2+1}}\right|+\log \beta\left(m_{1}, u, 2, k\right)+\log \beta\left(m_{2}, u, 2, k\right) \leq \log E_{m_{1}, m_{2}}(\varphi, \beta(u, 2, k))
$$

Again (3.6) taking into account in above inequality, we obtain

$$
\lim _{m_{1}+m_{2} \rightarrow \infty} \sup \frac{\log ^{[p-1]}\left(m_{1}+m_{2}\right)}{\log ^{[q]}\left[E_{m_{1}, m_{2}}(\varphi, \beta(u, 2, k))\right]^{\frac{-1}{\left(m_{1}+m_{2}\right)}}} \geq \lim _{m_{1}+m_{2} \rightarrow \infty} \sup \frac{\log ^{[p-1]}\left(m_{1}+m_{2}\right)}{\log ^{[q]}\left[a_{m_{1}, m_{2}}\right]^{\frac{-1}{\left(m_{1}+m_{2}\right)}}}
$$

Now using Lemma (2.1), since $\rho(p, q) \geq 1$ for $p=q$ the inequality for $p=q \geq 3$ gives

$$
\rho(p, q) \leq \max \left(1, L^{\prime}(p, q)\right)
$$

and for $p>q$ it gives

$$
\rho(p, q) \leq L^{\prime}(p, q)
$$

Hence combining above results we get $\rho(p, q) \leq P\left(L^{\prime}(p, q)\right)$

This is the proof of first step.

Now we consider the space $\beta(u, v, k) \neq 2$ we have

$$
E_{m_{1}, m_{2}}(\varphi, \beta(u, v, k)) \leq\left\|\varphi-H_{m_{1}, m_{2}}(\varphi)\right\|_{u, v, k}
$$

$=\left\{\int_{0}^{1} \int_{0}^{1}\left\{\left(1-r_{1}\right)\left(1-r_{2}\right)\right\}^{k\left(\frac{1}{u}-\frac{1}{v}\right)-1}\left(\sum_{i_{1}} \sum_{i_{2}} r_{1}^{2 i_{1}} r_{2}^{2 i_{2}}\left|a_{i_{1}, i_{2}}\right|^{2}\right)^{\frac{k}{v}} d r_{1} d r_{2}\right\}^{\frac{1}{k}}$

Where

$\sum_{i_{1}} \sum_{i_{2}} r_{1}^{2 i_{1}} r_{2}^{2 i_{2}}\left|a_{i_{1}, i_{2}}\right|^{v}=R_{1}+R_{2}+\sum_{i_{1}=m_{1}+1}^{\infty} \sum_{i_{2}=m_{2}+1}^{\infty} r_{1}^{2 i_{1}} r_{2}^{2 i_{2}}\left|a_{i_{1}, i_{2}}\right|^{v}$,

$R_{1}=\sum_{i_{1}=0}^{m_{1}} \sum_{i_{2}=m_{2}+1}^{\infty} r_{1}^{2 i_{1}} r_{2}^{2 i_{2}}\left|a_{i_{1}, i_{2}}\right|^{v}$ and $\quad R_{2}=\sum_{i_{1}=m_{1}+1}^{\infty} \sum_{i_{2}=0}^{m_{2}} r_{1}^{2 i_{1}} r_{2}^{2 i_{2}}\left|a_{i_{1}, i_{2}}\right|^{v}$

Since $R_{1}, R_{2}$ are bounded and $r_{1}, r_{2}<1$, therefore (3.11) becomes

$E_{m_{1}, m_{2}}(\varphi, \beta(u, v, k)) \leq C^{\prime \prime}\left[\int_{0}^{1}\left\{(1-r)^{k\left(\frac{1}{u}-\frac{1}{v}\right)-1}\right\} r^{(s+1) k} d r\right]\left\{\sum_{i_{1}=m_{1}+1}^{\infty} \sum_{i_{2}=m_{2}+1}^{\infty}\left|a_{i_{1}, i_{2}}\right|^{v}\right\}^{\frac{1}{v}}$

Where 


$$
\begin{aligned}
& \left\{\int_{0}^{1}\left\{(1-r)^{k\left(\frac{1}{u}-\frac{1}{v}\right)-1}\right\} r^{(s+1) k} d r\right\} \\
& =\left\{\int_{0}^{1}\left\{\left(1-r_{1}\right)^{k\left(\frac{1}{u}-\frac{1}{v}\right)-1}\right\} r_{1}^{\left(m_{1}+1\right) k} d r_{1}\right\}\left\{\int_{0}^{1}\left\{\left(1-r_{2}\right)^{k\left(\frac{1}{u}-\frac{1}{v}\right)-1}\right\} r_{2}{ }^{\left(m_{2}+1\right) k} d r_{2}\right\}
\end{aligned}
$$

Therefore

$$
E_{m_{1}, m_{2}}(\varphi, \beta(u, v, k)) \leq C^{\prime \prime} \beta\left(m_{1}, u, v, k\right) \beta\left(m_{2}, u, v, k\right)\left\{\sum_{i_{1}=m_{1}+1}^{\infty} \sum_{i_{2}=m_{2}+1}^{\infty}\left|a_{i_{1}, i_{2}}\right|^{v}\right\}^{\frac{1}{v}},
$$

Where $C^{\prime \prime}$ is constant and $\beta(m, u, v, k)$ is Euler's integral of the first kind. By using (3.2) we have

$$
\begin{gathered}
\sum_{i_{1}=m_{1}+1}^{\infty} \sum_{i_{2}=m_{2}+1}^{\infty}\left|a_{i_{1}, i_{2}}\right|^{v} \leq \sum_{i_{1}=m_{1}+1}^{\infty} \sum_{i_{2}=m_{2}+1}^{\infty}\left\{\exp ^{[q-1]}\left\{\log ^{[p-2]}\left(i_{1}+i_{2}\right)\right\}^{\frac{1}{\dot{L}^{(p, q)+\varepsilon}}}\right\}^{-v\left(i_{1}+i_{2}\right)} \\
=O(1)\left[\exp ^{[q-1]}\left\{\log ^{[p-2]}\left(m_{1}+m_{2}+2\right)\right\}^{\frac{1}{\dot{L}^{(p, q)+\varepsilon}}}\right]^{-v\left(m_{1}+m_{2}+2\right)}
\end{gathered}
$$

using this inequality in (3.12), we get

$$
\begin{aligned}
E_{m_{1}, m_{2}}(\varphi, \beta(u, v, k)) \leq & C^{\prime \prime} \beta\left(m_{1}, u, v, k\right) \beta\left(m_{2}, u, v, k\right) \times \\
& {\left[\exp ^{[q-1]}\left\{\log ^{[p-2]}\left(m_{1}+m_{2}+2\right)\right\}^{\frac{1}{L^{\prime}(p, q)+\varepsilon}}\right]^{-v\left(m_{1}+m_{2}+2\right)} }
\end{aligned}
$$

For $(p, q)=(2,1)$ the result has been proved by Ganti and Srivastava [2] .

Now $(p, q)=(2,2)$ we have from (3.13) that

$$
\begin{aligned}
& E_{m_{1}, m_{2}}(\varphi, \beta(u, v, k)) \leq C^{\prime \prime} \beta\left(m_{1}, u, v, k\right) \beta\left(m_{2}, u, v, k\right) \times\left\{\exp \left(m_{1}+m_{2}+2\right)^{\frac{1}{L^{\prime}(2,2)+\varepsilon}}\right\}^{-\left(m_{1}+m_{2}+2\right)} \\
& \text { Or }
\end{aligned}
$$

$\log E_{m_{1}, m_{2}}(\varphi, \beta(u, v, k))-\log \beta\left(m_{1}, u, v, k\right)-\log \beta\left(m_{2}, u, v, k\right)$

$$
\leq \log \left\{\exp \left(m_{1}+m_{2}+2\right)^{\frac{1}{L(2,2)+\varepsilon}}\right\}^{-\left(m_{1}+m_{2}+2\right)}
$$

Or

$$
\begin{array}{r}
\frac{1}{\log E_{m_{1}, m_{2}}(\varphi, \beta(u, v, k))-\log \beta\left(m_{1}, u, v, k\right)-\log \beta\left(m_{2}, u, v, k\right)} \\
\geq \frac{1}{-\left(m_{1}+m_{2}+2\right) \log \left\{\exp \left(m_{1}+m_{2}+2\right)^{\frac{1}{L^{\prime(2,2)+\varepsilon}}}\right\}}
\end{array}
$$

Since

and

$$
\beta\left[(n+1) k+1, k\left(\frac{1}{u}-\frac{1}{v}\right)\right]=\frac{\Gamma(n+1)(k+1) \Gamma\left(k\left(\frac{1}{u}-\frac{1}{v}\right)\right)}{\Gamma\left((n+1)(k+1)+k\left(\frac{1}{u}-\frac{1}{v}\right)\right)}
$$

$$
\left\{\beta\left[(n+1) k+1, k\left(\frac{1}{u}-\frac{1}{v}\right)\right]\right\}^{\frac{1}{(n+1)}} \cong 1 .
$$

Therefore from above inequality, we get

Or

$$
\frac{1}{\log E_{m_{1}, m_{2}}(\varphi, \beta(u, v, k))} \geq \frac{1}{-\left(m_{1}+m_{2}\right) \log \left\{\exp \left(m_{1}+m_{2}\right)^{\frac{1}{L(2,2)+\varepsilon}}\right\}}
$$


Or

$$
\log E_{m_{1}, m_{2}}(\varphi, \beta(u, v, k)) \leq \log \left\{\exp \left(m_{1}+m_{2}\right)^{\frac{1}{L^{\prime}(2,2)+\varepsilon}}\right\}^{-\left(m_{1}+m_{2}\right)}
$$

$$
\log \left[E_{m_{1}, m_{2}}(\varphi, \beta(u, v, k))\right]^{-\frac{1}{\left(m_{1}+m_{2}\right)}} \geq\left(m_{1}+m_{2}\right)^{\frac{1}{L^{\prime}(2,2)+\varepsilon}}
$$

Or

Proceeding to limits, we get

$$
L^{\prime}(2,2)+\varepsilon \geq \frac{\log \left(m_{1}+m_{2}\right)}{\log ^{[2]}\left[E_{m_{1}, m_{2}}(\varphi, \beta(u, v, k))\right]^{-\frac{1}{\left(m_{1}+m_{2}\right)}}}
$$

$$
L^{\prime}(2,2) \geq \lim _{m_{1}, m_{2} \rightarrow \infty} \sup \frac{\log \left(m_{1}+m_{2}\right)}{\log ^{[2]}\left[E_{m_{1}, m_{2}}(\varphi, \beta(u, v, k))\right]^{-\frac{1}{\left(m_{1}+m_{2}\right)}}}
$$

Or

$$
P\left(L^{\prime}(2,2)\right)-1 \geq \lim _{m_{1}, m_{2} \rightarrow \infty} \sup \frac{\log \left(m_{1}+m_{2}\right)}{\log ^{[2]}\left[E_{m_{1}, m_{2}}(\varphi, \beta(u, v, k))\right]^{-\frac{1}{\left(m_{1}+m_{2}\right)}}}
$$

Or

$$
P\left(L^{\prime}(2,2)\right)-1 \geq L^{\prime}(2,2)
$$

Or

$$
P\left(L^{\prime}(2,2)\right) \geq L^{\prime}(2,2)+1 \quad .
$$

Now consider the case $(p, q) \neq(2,1)$ and $(2,2)$ from $(3.13)$ we get $\log E_{m_{1}, m_{2}}(\varphi, \beta(u, v, k))-\log \beta\left(m_{1}, u, v, k\right)-\log \beta\left(m_{2}, u, v, k\right)-\log C^{\prime \prime}$

Using (3.14), we obtain

$$
\leq \log \left[\exp ^{[q-1]}\left\{\log ^{[p-2]}\left(m_{1}+m_{2}+2\right)\right\}^{\frac{1}{L^{\prime}(p, q)+\varepsilon}}\right]^{-\left(m_{1}+m_{2}+2\right)}
$$

$$
\frac{1}{\log E_{m_{1}, m_{2}}(\varphi, \beta(u, v, k))} \geq \frac{1}{-\left(m_{1}+m_{2}\right) \log \left[\exp ^{[q-1]}\left\{\log ^{[p-2]}\left(m_{1}+m_{2}\right)\right\}^{\frac{1}{L^{(p, q)+\varepsilon}}}\right]}
$$

Or

Or

$$
\left[\log E_{m_{1}, m_{2}}(\varphi, \beta(u, v, k))\right]^{-\frac{1}{\left(m_{1}+m_{2}\right)}} \geq \exp ^{[q-1]}\left\{\log ^{[p-2]}\left(m_{1}+m_{2}\right)\right\}^{\frac{1}{L^{(p, q)+\varepsilon}}}
$$

Proceeding to limits, immediately we get

$$
L^{\prime}(p, q)+\varepsilon \geq \frac{\log ^{[p-1]}\left(m_{1}+m_{2}\right)}{\log [q]\left[E_{m_{1}, m_{2}}(\varphi, \beta(u, v, k))\right]^{-\frac{1}{\left(m_{1}+m_{2}\right)}}}
$$

$$
\rho(p, q) \geq P\left(L^{\prime}(p, q)\right)
$$

To prove reverse inequality taking (3.12) into account this gives

$$
\left|a_{m_{1}+1, m_{2}+1}\right| \beta\left(m_{1}, u, v, k\right) \beta\left(m_{2}, u, v, k\right) \leq E_{m_{1}, m_{2}}(\varphi, \beta(u, v, k))
$$

Or

$$
-\log E_{m_{1}, m_{2}}(\varphi, \beta(u, v, k)) \leq-\log \left|a_{m_{1}+1, m_{2}+1}\right|-\log \beta\left(m_{1}, u, v, k\right)-\log \beta\left(m_{2}, u, v, k\right)
$$

Again using (3.14), we get

$$
\frac{\log \left(m_{1}+m_{2}\right)}{\log ^{[2]}\left[E_{m_{1}, m_{2}}(\varphi, \beta(u, v, k)]^{-\frac{1}{\left(m_{1}+m_{2}\right)}}\right.} \geq \frac{\log \left(m_{1}+m_{2}\right)}{\log ^{[2]}\left|a_{m_{1}+1, m_{2}+1}\right|^{-\frac{1}{\left(m_{1}+m_{2}\right)}}}
$$

In view of lemma (2.1), we obtain

$$
L^{\prime}(2,2) \geq \rho(2,2)-1
$$


Or

$$
L^{\prime}(2,2)+1 \geq \rho(2,2)
$$

Since $\rho(p, q) \geq 1$ for $p=q$ the inequality for $p=q \geq 3$ gives $\rho(p, p) \leq \max \left(1, L^{\prime}(p, p)\right)$ and for $p>q$ it gives

$$
\rho(p, q) \leq L^{\prime}(p, q)
$$

Combining (3.15), (3.16), (3.17) and (3.18) the proof of second step is immediate.

Now consider the third step. Let $0<u<v<2$ and $k, v \geq 1$.

Since

$$
E_{m_{1}, m_{2}}\left(\varphi, \beta\left(u_{1}, v_{1}, k_{1}\right)\right) \leq 2^{\left(\frac{1}{u_{1}}-\frac{1}{v_{1}}\right)}\left[k\left(\left(\frac{1}{u}-\frac{1}{v}\right)\right)\right]^{\left(\frac{1}{u_{1}}-\frac{1}{v_{1}}\right)} E_{m_{1}, m_{2}}(\varphi, \beta(u, v, k))
$$

where $u_{1}=u, v_{1}=2$ and $k_{1}=k$ and the condition (3.1) is already proved for the space $\beta(u, 2, k)$ hence

$$
\begin{aligned}
\lim _{m_{1}+m_{2} \rightarrow \infty} \sup \frac{\log ^{[p-1]}\left\{\left(m_{1}+m_{2}\right) a_{m_{1} 1, m_{2}}\right\}}{\log ^{[q]}\left[E_{m_{1}, m_{2}}(\varphi, \beta(u, v, k))\right]^{\frac{-1}{\left.m_{1}+m_{2}\right)}}} \\
\quad \geq \lim _{m_{1}+m_{2} \rightarrow \infty} \sup \frac{\log ^{[p-1]}\left\{\left(m_{1}+m_{2}\right) a_{m_{1} 1, m_{2}}\right\}}{\log [q]\left[E_{m_{1}, m_{2}}(\varphi, \beta(u, 2, k))\right]^{\frac{-1}{\left(m_{1}+m_{2}\right)}}}
\end{aligned}
$$

Now let $0<u \leq 2<v$.Since

$$
M_{1}\left(\varphi, r_{1}, r_{2}\right) \leq M_{2}\left(\varphi, r_{1}, r_{2}\right), 0<r_{1}<r_{2}<1 \text {. }
$$

Therefore

$$
\begin{gathered}
E_{m_{1}, m_{2}}\left(\varphi, \beta\left(u_{1}, v_{1}, k_{1}\right)\right) \geq\left\{\int_{0}^{1} \int_{0}^{1}\left\{\left(1-r_{1}\right)\left(1-r_{2}\right)\right\}^{k\left(\frac{1}{u}-\frac{1}{v}\right)-1} S d r_{1} d r_{2}\right\} \\
\geq\left[\left|a_{m_{1}, m_{2}}\right| \beta\left(m_{1}, u, v, k\right) \beta\left(m_{2}, u, v, k\right)\right]
\end{gathered}
$$

16.

where

$S=\inf \left[M_{2}^{k}\left(\varphi-u, r_{1}, r_{2}\right): p \in P\right]$. Hence we get

$$
\begin{aligned}
\lim _{m_{1}+m_{2} \rightarrow \infty} \sup \frac{\log ^{[p-1]}\left\{\left(m_{1}+m_{2}\right) a_{m_{1} 1, m_{2}}\right\}}{\log ^{[q]}\left[E_{m_{1}, m_{2}}(\varphi, \beta(u, v, k))\right]^{\frac{-1}{\left(m_{1}+m_{2}\right)}}} & \geq \lim _{m_{1}+m_{2} \rightarrow \infty} \sup \frac{\log ^{[p-1]}\left\{\left(m_{1}+m_{2}\right) a_{m_{1} 1, m_{2}}\right\}}{\log { }^{[q]}\left[\left|a_{m_{1}, m_{2}}\right|\right]^{\left(m_{1}+m_{2}\right)}}
\end{aligned}
$$

In view of (3.19), (3.20) and Lemma 2.1, we get the required result. This is complete proof of the theorem.

Theorem 3.2. If $\varphi\left(z_{1 .} z_{2}\right)=\sum_{m_{1}, m_{2}=0}^{\infty}\left\{a_{m_{1}, m_{2}}\left(z_{1}^{m_{1}}, z_{2}^{m_{2}}\right)\right\}$ be an entire function, then for a pair of integers $(p, q), p \geq 2, q \geq 1$ the function $\varphi\left(z_{1 .} z_{2}\right) \in H$ is of $(p, q)$-order $\rho(p, q)$ if and only if $\quad \rho(p, q)=$ $P\left(L^{* *}(p, q)\right)$

where

$$
L^{* *}(p, q)=\lim _{m_{1}+m_{2} \rightarrow \infty} \sup \frac{\log ^{[p-1]}\left\{\left(m_{1}+m_{2}\right) a_{m_{1}, m_{2}}\right\}}{\log ^{[q-1]}\left\{\frac{1}{\left(m_{1}+m_{2}\right)} \log \left[E_{m_{1}, m_{2}}\left(\varphi, H_{\vartheta}\right)\right]^{-1}\right\}} .
$$

Proof. Let $\varphi\left(z_{1} . z_{2}\right)=\sum_{m_{1}, m_{2}=0}^{\infty}\left\{a_{m_{1}, m_{2}}\left(z_{1}^{m_{1}}, z_{2}^{m_{2}}\right)\right\} \in H_{\vartheta}$ be an entire transcendental function. Since $\varphi$ is entire, we have

and $\varphi \in H_{\vartheta}$, therefore

$$
\lim _{m_{1}, m_{2} \rightarrow \infty}\left|a_{m_{1}, m_{2}}\right|^{\frac{1}{\left(m_{1}+m_{2}\right)}}=0
$$

$$
M_{\vartheta}\left(\varphi, r_{1}, r_{2}\right)<\infty,
$$

and $\varphi\left(z_{1} . z_{2}\right) \in \beta(u, v, k), 0<u<v \leq \infty, k \geq 1 . B y(1.1)$ we have

$$
E_{m_{1}, m_{2}}(\varphi, \beta(v / 2, v, v)) \leq K E_{m_{1}, m_{2}}\left(\varphi, H_{\vartheta}\right), 1 \leq v<\infty,
$$

where $\mathrm{K}$ is a constant independent of $m_{1}, m_{2}$ and $\varphi$ In the case of space $H_{\infty}$, 
From (3.23) we have

$$
E_{m_{1}, m_{2}}(\varphi, \beta(u, \infty, \infty)) \leq E_{m_{1}, m_{2}}\left(\varphi, H_{\vartheta}\right), 0<u<\infty .
$$

$$
\begin{gathered}
\xi(\varphi)=\lim _{m_{1}+m_{2} \rightarrow \infty} \sup \frac{\log ^{[p-1]}\left\{\left(m_{1}+m_{2}\right) a_{m_{1}, m_{2}}\right\}}{\log [q-1]\left\{\frac{1}{\left(m_{1}+m_{2}\right)} \log \left[E_{m_{1}, m_{2}}\left(\varphi, H_{\vartheta}\right)\right]^{-1}\right\}} \\
\geq \lim _{m_{1}+m_{2} \rightarrow \infty} \sup \frac{\log ^{[p-1]}\left\{\left(m_{1}+m_{2}\right) a_{m_{1}, m_{2}}\right\}}{\log ^{[q-1]}\left\{\frac{1}{\left(m_{1}+m_{2}\right)} \log \left[E_{m_{1}, m_{2}}(\varphi, \beta(v / 2, v, v))\right]^{-1}\right\}} \\
\geq L^{* *}(p, q), 1 \leq v \leq \infty,
\end{gathered}
$$

using (3.24) we prove inequality (3.25) for the case $v=\infty$.

For the reverse inequality, we have

$$
E_{m_{1}, m_{2}}\left(\varphi, H_{\vartheta}\right) \leq O(1) \sum_{i_{1}=m_{1}+1}^{\infty} \sum_{i_{2}=m_{2}+1}^{\infty}\left|a_{i_{1}, i_{2}}\right|
$$

using (3.2) we have

Or

$$
E_{m_{1}, m_{2}}\left(\varphi, H_{\vartheta}\right) \leq O(1) \sum_{i_{1}=m_{1}+1}^{\infty} \sum_{i_{2}=m_{2}+1}^{\infty}\left\{\exp ^{[q-1]}\left\{\log ^{[p-2]}\left(i_{1}+i_{2}\right) a_{i_{1}, i_{2}}\right\}^{\frac{1}{L^{\prime}(p, q)+\varepsilon}}\right\}^{-2\left(i_{1}+i_{2}\right)}
$$

$$
E_{m_{1}, m_{2}}\left(\varphi, H_{\vartheta}\right) \leq O(1)\left[\exp ^{[q-1]}\left\{\log ^{[p-2]}\left(i_{1}+i_{2}\right) a_{i_{1}, i_{2}}\right\}^{\frac{1}{L^{\prime}(p, q)+\varepsilon}}\right]^{-2\left(m_{1}+1+m_{2}+1\right)}
$$

which gives

$$
L^{\prime}(p, q)+\varepsilon \geq \frac{\log ^{[p-1]}\left\{\left(m_{1}+m_{2}\right) a_{m_{1}, m_{2}}\right\}}{\log ^{[q]}\left[E_{m_{1}, m_{2}}\left(\varphi, H_{\vartheta}\right)\right]^{\frac{-1}{\left(m_{1}+m_{2}\right)}}}
$$

Proceeding to limits we get

$$
L^{\prime}(p, q) \geq \xi(\varphi)
$$

In the consequence of Theorem 3.1 with (3.25) and (3.26) we obtain the result immediately. Now to prove sufficiency, assume that the condition (3.21) is satisfied. Then it follows that

$$
\log \left[\frac{1}{E_{m_{1}, m_{2}}\left(\varphi, H_{\vartheta}\right)}\right]^{\frac{1}{\left(m_{1}+m_{2}\right)}} \rightarrow \infty \text { as } m_{1}+m_{2} \rightarrow \infty
$$

It gives

$$
\lim _{m_{1}+m_{2} \rightarrow \infty}\left[\frac{1}{E_{m_{1}, m_{2}}\left(\varphi, H_{\vartheta}\right)}\right]^{\frac{1}{\left(m_{1}+m_{2}\right)}}=0 .
$$

This relation and the estimate $\left|a_{m_{1}, m_{2}}(\varphi)\right| \leq E_{m_{1}, m_{2}}\left(\varphi, H_{\vartheta}\right)$ yield the relation (3.22). It follows that $\varphi\left(z_{1 .} z_{2}\right) \in$ $H_{\vartheta}$ is an entire transcendental function.

Hence the proof is completed.

\section{REFERENCES}

[1] Bose, S.K. and Sharma, D., Integral functions of two complex variables, Compositio Math. 15 (1963), $210-226$.

[2] Ganti, R. and Srivastava, G.S., Approximation of entire functions of two complex variables in Banach spaces, J. Inequalities in Pure \& Applied Mathematics, 7, Issue 2, (2006), 1-11.

[3] Juneja, O.P., Kapoor, G.P. and Bajpai, S.K., On the $(p, q)$ - order and lower $(p, q)-$ order of an entire function, J. Reine Angew. Math. 282 (1976), 53-67.

[4] Vakarchuk, S.B. and Zhir, S.I., On some problems of polynomial approximation of entire transcendental functions, Ukarainian Mathematical Journal, (54) (9) (2002), 1393-1401. 